\title{
Aircraft Maintenance Planning Using Fuzzy Critical Path Analysis
}

\author{
Omer Atli $^{*}$ \\ Aeronautics and Space Technologies Institute \\ Turkish Air Force Academy \\ Yesilyurt, Bakirkoy, Istanbul 34149, Turkey \\ Cengiz Kahraman ${ }^{\dagger}$ \\ Department of Industrial Engineering, Istanbul Technical University \\ Istanbul Turkey \\ E-mail:kahramanc@itu.edu.tr \\ Received 1 August 2011 \\ Accepted 1 April 2012
}

\begin{abstract}
Aircraft's availability is certainly one of the most important features of modern avionic industry. The aircraft maintenance scheduling is one of the major decisions an airline has to make during its operation. When an aircraft maintenance event occurs, the overhaul tasks management process requires the execution of all tasks to perform and has to guarantee the on-time aircraft delivery and the respect of the daily flight schedule. Though maintenance scheduling comes as an end stage in an airline operation, it has potential for cost savings. Maintenance scheduling is an easily understood but difficult to solve problem. Given a flight schedule with aircraft assigned to it, the aircraft maintenance-scheduling problem is to determine which aircraft should fly which segment and when and where each aircraft should undergo different levels of maintenance check. The objective of this paper is to minimize the aircraft maintenance planning time and to show how to create a plan with critical path analyses under fuzzy environment. We use trapezoidal fuzzy numbers for activity times and Activity-on-Node (AON) representation in fuzzy critical path method (FCPM). An illustrative example is given for Aircraft Gas Turbine Engine Repair/Overhaul problem.
\end{abstract}

Keywords: Fuzzy critical path analysis, Fuzzy set theory, Aircraft maintenance planning, scheduling.

\section{Introduction}

Planning and scheduling functions are the key deliverables of the planning role. This is where the most gains in execution have the potential to be made and acted upon. In some larger organizations these are split, allowing more adequate resources for each role. The role of the planner needs to cover the full range of the work order system, from input into coding, prioritization and a degree of autonomy in execution. As such these roles, more and more need to be staffed by skilled and versatile people.

Maintenance management is a strategic process for airlines, both for quality and economy, since it must achieve and satisfy -at the same time- goals related to safety levels, to the aircrafts operating availability, to the on-time reliability of scheduled flight and to the

\footnotetext{
*Corresponding author: atliomer@ gmail.com Tel: +90212 6632490.

${ }^{\dagger}$ Tel: +902122931300/ 2035.
} 
costs. A correct maintenance management concurs to the systems efficiency by

- Reducing at the minimum the maintenance duration time, therefore containing the aircraft dwell time (grounding). This allows increasing the efficiency related to the operating costs and the possibility to offer low rates to the customers.

- Reducing the delays on the aircraft delivery dates. This occurs to decrease the activity delay causes and to assure the on-time scheduled flight to the customers.

The important issue is maintenance and safeties, aircraft safety levels guaranteed by technical maintenance are fundamental for air transport safety. It's because of maintenance effectiveness and efficiency that since last decades aircraft accidents due to the technical problems have been remarkably reduced. All the parts and components of aircrafts have or might have a time limit on the employment within which they must be inspected.

Aircraft maintenance is a very important aspect of aircraft fleet management since it usually accounts for a substantial part of the overall operational costs and sets constraints on the planning of flight operations. Maintenance scheduling is typically subjected to a large number of constraints. Among them are capacities of maintenance facilities, capacities and skills of maintenance workers, maintenance rules and flight plans. Moreover, the costs of a specific maintenance action are not generally constant but may depend both on time and on the sequence of maintenance actions to be performed on a single aircraft or an entire fleet. For example, maintenance costs can often be reduced by performing several maintenance actions on one occasion. Due to the number of constraints to be observed and the complexity of costs, maintenance scheduling problems are notoriously difficult and timeconsuming to solve, especially for large fleets. Additionally, maintenance plans, even for fleets servicing scheduled flights are hard to follow due to changes in flight plans or corrective maintenance actions on aircraft components. This is even truer for fleets servicing unscheduled flights such as ambulance, rescue, leisure, charter and air force flights. Fleets allocated for such flights are typically facing highly variable and unpredictable demand. Therefore maintenance plans have to be updated frequently. Under such conditions, maintenance scheduling can become very tedious, if carried out in a non-automated way.

In reality, it is often difficult to obtain estimates of activity time, due to the uncertainty of information as well as variation in the management scenario of aircraft maintenance planning. Moreover, linguistic terms such as "approximately between 1 and 2 man-hours" and "around 1.5 man-hours" are frequently used to estimate maintenance planning times. These conventional approaches, both deterministic and random process, tend to be less effective in maintenance planning project the imprecise or vague nature of these linguistic assessments. The fuzzy set theory can play a significant role in this kind of decision-making environment.

The constructed network is an aid in the control of actual project implementation. The method, however, requires the availability of deterministic time duration or probability distribution function for each activity. This requirement is difficult to fulfill in a project where many of the activities will be executed for the first time. In this case, more often than not, there is a vague idea about activity duration which then has to be estimated subjectively.

In today's highly competitive business environment, project management's ability to schedule activities and control progress within cost, time, and quality is becoming important to obtain competitive priorities such as on-time delivery and customization. When the activity times in the project are deterministic and known, critical path method (CPM) has been demonstrated to be a useful tool in managing projects in an efficient manner to meet this challenge. However, there are many cases where the activity times may not be presented in a precise manner. To solve these problems with imprecise data researchers have presented the program evaluation and review technique (PERT) and Monte Carlo simulation (Kenzo, 2002; Ragsdale, 1986), which are based on the probability theory. However, there are some critiques of CPM and PERT. In PERT there are some assumptions for simplifying the model, for example use of the beta distribution. Also to provide an appropriate distribution for activity times we need historical data. But in the real world some activities have never proceeded and we have to use human judgment instead of stochastic assumptions to determine activity times. The detailed critiques of PERT can be found in the paper of Shipley (Shipley et al., 1997). An alternative way to deal with imprecise is to use of the fuzzy set theory instead of probability theory. In this case activity times are represented by fuzzy numbers. The concept of fuzzy set was introduced by Zadeh in 1965, to provide a model where inexactness and imprecision arise. Fuzzy sets were then applied among others to real algebra, where the term fuzzy number was coined. A method that utilizes fuzzy sets in a network is presented by several researchers, for example, Dubois and Prade [2], Chanas and Kamburowski [5], Chanas [6], Buckley [7], Mares 
[8], Gazdik [9], McCahon and Lee [10], Tsujimura et al., [11], Chanas and Zielinski [12], Han et.al. [13], Liang and Han [14], Nasution [16, 17], and Slyeptsov and Tyshchuk [18].

In this paper we propose a mathematical model to deal with project scheduling problem under vagueness. It is supposed that durations of activities are imprecise and represented by trapezoidal fuzzy numbers. The objective of this paper is to model aircraft maintenance planning under fuzziness using critical path analysis.

The rest of the paper is organized as follows. In the section 2, maintenance planning is described. The third section briefly describes classical CPM scheduling in maintenance planning. In Section 4, we present a wide literature summary and gap analysis of fuzzy critical path analysis in maintenance planning. In the fifth section, an application of the Fuzzy CPM analysis method is explained. An illustrative example is given in order to show the application of the proposed models and algorithms and the foundations of the proposed solution approach are provided in the sixth section. Finally, in the seventh section, we present the computational fuzzy CPM algorithm's results; and concluding remarks are made, along with a discussion about further research.

\section{Maintenance Planning}

While maintenance has always been essential to aviation, its mission has matured along with the industry. A daily flight schedule is originated, assuring on one side the highest safety levels and on the other side, economical strategies, pursuing the goal of maximizing the aircraft use, while strictly adhering to the daily flight schedule; "on-time departure and minimum aircraft ground time are a target for the airline, while safety levels are constraints to respect". To achieve this goal, airlines must plan the maintenance operations placing them in strategic time between arrivals and departures, in order to minimize the aircraft grounding, to assure the on-time aircraft delivery and the respect of the daily flight schedule.

It is well known that the aircraft's availability, i.e. the probability of having the system ready for working, is surely one of the most important characteristic for the aircraft industry. In order to pursue the goal of increasing the aircraft's availability, either the logistic reliability or the maintainability (i.e. the mean time to repair) of the system can be augmented. As the complexity of modern systems inevitably implies a worse logistic reliability, one generally seeks the increase of maintainability.
The maintenance plan defines all scheduled maintenance tasks that have to be accomplished. They may be both simple activities, which have to be performed quite often (for instance, at the end of each flight), and demanding operations, which have to be carried out less frequently. However, as the number of aircraft's scheduled maintenance tasks is generally impressive, these activities are normally grouped together as sets of operations that have to be performed, for example every 100 flight hours. This implies a fixed number of various maintenance tasks, which may require different personnel's skills, as well as different equipment, and aircraft's conditions. Quite obviously, the problem of finding the maintenance tasks' scheduling, which minimizes the time and/or the cost of maintenance operations, arises.

Aircraft maintenance plans are becoming more sophisticated, and provide more flexibility for maintenance planners to decide when tasks should be performed. However, this can complicate the task of compiling work scopes for a hangar checks.

When the $\$ 100$ billion that airlines spend each year on aircraft maintenance, aircraft is taken into consideration, it is easy to see how good planning can contribute to cost control. Getting maintenance planning wrong can ultimately have disastrous consequences for airlines.

Regular maintenance and inspections ensure that aircraft are airworthy and prevent component and system failures during operation. There is always a tension within airlines between the need to maximize the availability of aircraft for operations and having sufficient downtime for maintenance. While commercial departments want to maximize aircraft utilization, maintenance departments need to have the aircraft available in the right locations for reasonable periods of time. Unexpected failures of components or a shortage of parts or mechanics with particular skills and qualifications add to the complexity and stress of planning maintenance. Many inputs need to be juggled by maintenance planners to produce a workable solution: the flight schedule; the aircraft technical status; material availability; hangar space; mechanics' availability; and the scheduled tasks themselves.

The cost savings derived from efficient maintenance planning, however, can be substantial. Out of a fleet of 20 or 30 aircraft there will always be three or four aircrafts in maintenance and out of revenue service. If maintenance planning can be optimized, and hangar time reduced, it might be possible to release one of those aircrafts into service and reduce the number down for maintenance to two or three. 
When maintenance planning is this efficient, it produces a 'free' aircraft to generate additional capacity and revenue, or reduce the fleet requirement by one. The scope for this level of efficiency is naturally higher with larger fleets. At a micro level there are still substantial savings to be realized, for example task yield: the actual interval over which the task is accomplished compared to its theoretical allowable interval, expressed as a percentage.

When all the regular tasks are taken into consideration it is easy to see how well planning can save a lot of money. It is also equally vital for maintenance planning that planners do not mistimed a task and exceed the allowable interval. Grounding the airline for safety reasons can be even more catastrophic. It is a fine line to walk between safety and optimum economy."

Aircraft maintenance takes place in a series of checks of increasing diligence with the exception of unscheduled fixes. The frequency of these checks depends on the combination of flight hours and number of take-off and landing cycles, and may be performed at any site appropriately equipped. Because each aircraft type has different inventory requirement, little savings can be achieved by combining facilities for different fleets. To be in compliance with the Federal Aviation Administration (FAA) constraints, some companies have adopted maintenance policies that call for routine inspections at least every four days. There are four major types of check mandated by the FAA that each aircraft has to undergo. These vary in scope, duration, and frequency. So, there are several ways for classifying maintenance. There are two general types of maintenance performed without distinction as to levels of maintenance. They are upkeep and overhaul.

Upkeep maintenance is preventive, corrective, or additive maintenance performed by catapult and arresting gear crew members. It includes servicing, periodic inspection, functional and bench testing, replacement, preservation, and repair of catapult and arresting gear equipment.

Overhaul maintenance is the process of disassembly sufficient to inspect all the operating components. It includes the repair, replacement, or servicing as necessary followed by reassembly and functional testing. Upon completion of the overhaul process the equipment will be capable of performing its intended service. Much of this work is normally done at naval overhaul and depot facilities, contractor plants, and other industrial facilities.

The goal of a maintenance action is to restore and to maintain the aircraft ability to work, delaying the aging process and preventing or correcting possible breakdowns.

A maintenance action can be executed before the breakdown (preventive maintenance), with the scope to avoid it or to delay it, or after the breakdown (corrective maintenance); in order to remove it maintenance can be classified by both product type (airframe, engine and components) and the timing and purpose of the work. The latter yields four grouping scheduled maintenance, (such as airframe and engine check) non-routine maintenance, refurbishments and modifications. Another way to classify maintenance-widely accepted from aeronautics authorities distinguishes;

Line maintenance performed at the airport line station some time even on the ramp; these operations have an expiration date independent from the aircraft type and from a prefixed maintenance cycle.

- Pre-flight check, post-flight check transit check in connection to the specific flight

- Daily check, weekly check belong to the line maintenance

Base maintenance performed at the main base inside the hangar, these operations are related to a specific maintenance cycle, with a periodic expiration based on aircraft type and on number of flight hours or cycles; the routine maintenance operations, classified in overhauls and inspections according to the operations weight, and referred to as "checks" or "letter type checks" belong to the base maintenance. "Type A check" is the form that most routine maintenance takes and might have several grades- type A check through type D check- according to be related job list gradually increasing-all performed at regular intervals.

Light maintenance, line maintenance, overnight maintenance, Type A and B checks are instances of light maintenance; for line maintenance works are managed at the airport; overnight, in the allotted time, not to disrupt the aircraft's flight schedule;

Heavy maintenance involves type $\mathrm{C}$ checks and type $\mathrm{D}$ checks. For a complete comprehension of the problem, we report a shortly description of "letter checks" meaning

"Type A Check" is the most frequent check and can be carried out between 450 and 650 flight hours, depending on the aircraft model. More than 400 tasks are performed on the aircraft.

"Type B Check" is performed every 1950 flight hours. It includes on Type A check and the aircraft remains under inspection for about 4 days.

"Type C Check" is fundamental air worthiness inspection scheduled at time intervals of 15 to 18 months and it requires from 8 to 15 working days. It 
includes both "Type A check" and "type B check" and in addition, other activities are performed. It includes also a post check flight test.

"Type D check" or "RED" is the most intensive form of routine maintenance and it is carried out every 6 to 8 years. It is very heavy inspection and the aircraft is essentially stripped to its shell and rebuilt with the goal of returning it to the original (zero timed) condition as much as possible. The maintenance tasks to be performed are in great number (2000-3000) and the whole process required from one to two months durations.

\section{Classical CPM Scheduling in Maintenance Planning}

One of the more important tools available to the scheduler during a maintenance outage is the Critical Path Method (CPM) of scheduling. Whether computer automated or performed manually, CPM is the quickest and most accurate method available for scheduling and managing large, complex work packages, optimizing schedules and for modifying schedules when disruptions occur. CPM scheduling is a graphical technique used for illustrating activity sequences, together with each activity's expected duration, to portray project execution steps in precedence order.

During many large overhaul and outage situations, a project or job may consist of a number of activities that can be carried out simultaneously. The effect of doing several activities at the same time is to reduce the total time for the job to be completed. The total man-hours involved will however remain substantially the same. By tracing the various work element paths from project start to project completion, the most time-consuming path is the length of time it will take to complete the job. It is identified as the critical path because any delays along that path will delay the entire project.

Development of a CPM schedule begins by representing the project graphically by a network built up from either circles or squares and lines or arrows, which lead up to or emerge from the circles or squares. Depending on the method used, the circles or squares represent either activities or events (the completion of an activity). Connecting the circles or squares with lines or arrows represents a sequence of activities in which each one is dependent on the previous one. In other words, one activity must be completed in order to begin the next activity. The initial network development is the most important and difficult in developing CPM schedules. Graphing out the job activities and dependencies to develop the network requires intimate knowledge of the constituent parts of the project. It is remarkable how many projects are undertaken which have not been "thought through" and many persons undertaking CPM for the first time are astonished at their own ignorance of the project they are planning.

CPM utilizes the Activity-on-Node (AON) representation as opposed to the PERT Activity-on-Arc (AOA) structure. In this study we used AON representation. The characteristics of these two representations are:

\section{AON representation:}

- Each activity is represented by a node in the network.

- A precedence relationship between two activities is represented by an arc or link between the two.

- AON may be less error prone because it does not need "dummy" activities or arcs.

\section{AOA representation:}

- Each activity is represented by an arc in the network.

- If activities A and B must precedence activity C, there are two arcs, $\mathrm{A}$ and $\mathrm{B}$, leading into arc $\mathrm{C}$. Thus, the nodes, the points where $\operatorname{arcs} \mathrm{A}$ and $\mathrm{B}$ join arc $\mathrm{C}$, represent events or "milestones" rather than activities (e.g., "finished activities $\mathrm{A}$ and $\mathrm{B}$ "). Dummy activities of zero duration may be required to represent precedence relationships accurately.

- AOA historically has been more popular, perhaps because of its similarity to Gantt Chart Schedules used by most project management software.

The CPM technique provides for the computation of the network "critical path," which consists of the sequence of project activities that determine the minimum required project time. To identify the critical path, first three characteristics of each event are determined: the earliest expected start time $T_{A i}$ in the forward pass (from start toward end event); the latest allowable start time $T_{L i}$ in the backward pass (from end toward start event); and the slack (float) $s_{i}=T_{L i}-T_{A i}$ of each event $i$. The slack of an event $i$ is the possible delay in the realization of event $i$ that causes no delay in total project duration. The critical path is the path from start event to end event where the slacks of the events are all zeros. An increase in the duration of any activity along the critical path will certainly increase the total project duration. Beside event slack $S_{i}$, the slack can be calculated for activity $a=(i, j)$. Each of the events $i$ and $j$ of activity $(i, j)$ possesses two possible time values $T_{A i}, T_{L i}$ and $T_{A j}, T_{L j}$, respectively. Therefore four relations are possible. Two of them are the total slack and free slack. The total slack is calculated 
as $T_{L j}-\left(T_{A i}+t_{i j}\right)$; it is the amount of time the activity can be delayed without impacting the latest allowable start time of subsequent activities. The free slack is calculated as $T_{A j}-\left(T_{A i}+t_{i j}\right)$. The free slack is the amount of time the activity can be delayed without impacting the early start time of subsequent activities.

\section{Literature Review}

Fuzzy critical path analysis has been an attractive topic for many researchers for three decades. In the following, we briefly summarize the related literature. Chanas and Zielinski [12] propose a method to undertake critical path analysis (CPA) of the network with fuzzy activity times (interval activity times, fuzzy numbers of the L-R type) by directly applying the extension principle to the classical criticality notion treated as a function of activity duration time in the network.

The method that Chanas and Kamburowski [5] developed is analogous to CPM or PERT, but, because activity durations are fuzzy sets on time space, they called the method fuzzy PERT (FPERT). Its goal is to determine the fuzzy project completion time; thus there was no mention of backward pass and identification of a critical path. To calculate the earliest start time $T_{A i}$, of event $i$ using the extended maximum, Eq. (1) is used:

$T_{A i}=\max _{h}\left\{T_{A h} \oplus t_{h i}\right\}$, where $h \in \Gamma_{G}^{-}(i), T_{A s}=0$

where the set of all immediate predecessors of vertex $i$ is denoted by $\Gamma_{G}^{-}(i)$ and the earliest start time $T_{A s}$, of event s (start event).

Chanas [6] states that the fuzzified backward pass cannot be performed by choosing $T_{L e}=T_{A e}$, where $T_{A e}$ is determined according to (1). To execute the backward pass he proposes to initiate the calculation with independently fixed deterministic or fuzzy target time. He defines the degree of criticality of an event as follows. Let $\bar{T}_{A i}$ be the earliest start time of event $i$ calculated according to (1) but starting from the end event, that is,

$\bar{T}_{A i}=\max _{j}\left\{\bar{T}_{A j} \oplus t_{i j}\right\}, \quad$ where $j \in \Gamma_{G}^{+}(i), \bar{T}_{A e}=0$.

where the latest allowable start time $T_{L e}$, of event e (end event) in the backward pass (from end toward start event); the earliest expected start time $T_{A e}$, of event $\mathrm{e}$ (end event) in the forward pass (from start toward end event); the set of all immediate successors of $i$ is denoted $\Gamma_{G}^{+}(i)$.
He then defines the fuzzy set on the set of vertices of $\mathrm{C}$ with membership function

$$
\mu_{C}(i)=\operatorname{Poss}\left\{T_{A i} \oplus \bar{T}_{A i} \text { is } T_{A e}\right\}
$$

as the fuzzy set of critical events, where

$$
\begin{aligned}
& \operatorname{Poss}\{A \text { is } \mathrm{B}\}=\sup _{t}\{A \cap B\} \\
& =\sup \min \left\{\mu_{A}(t), \mu_{B}(t)\right\} .
\end{aligned}
$$

A fuzzy set $U C$ on the set $U$ of activities with membership function

$\mu_{U C}(i)=\operatorname{Poss}\left\{T_{A i} \oplus t_{i j} \oplus \bar{T}_{A i}\right.$ is $\left.T_{A e}\right\}$

is called a fuzzy critical activity.

A fuzzy set $P C$ on the set $P$ of paths with membership function

$\mu_{P C}(p)=\operatorname{Poss}\left\{T_{p}\right.$ is $\left.T_{A e}\right\}$,

where $T_{p}$ is the duration of the path $p \in P$ is called a fuzzy set of critical paths.

Dubois and Prade [2] calculate the latest allowable start time $T_{L i}$ in the backward pass, even though this is achieved by fixing a fuzzy value for end event $e, T_{N}$, different from $T_{A e}$. They argue that it is necessary to define $T_{N}$ independently of $T_{A e}$ in an imprecise environment because the imprecision must accumulate and there is a risk of counting it twice in the course of calculating the $T_{L i}$ starting with $T_{L e}=T_{A e}$.

Buckley [7] gives a definition of possibility distribution $T_{A i}$ for event $i$, slack, earliest start time, and latest start time of activity $(i, j)$. He gives an algorithm to compute the possibility distribution for project duration, $T_{A e}$ where durations have trapezoidal possibility distribution. The computation is done using ordinary CPM in one $\alpha$-level set of fuzzy time. The inputs are the lower bounds of durations in the particular $\alpha$-level set, this will give the lower bound of project duration. Then the computation is repeated for all the upper bounds and these results in the upper bound of the project duration. He then points out that the computation of the other three characteristics is far more difficult since we have to evaluate CPM $2^{W}$ times, where $W$ is the number of jobs or activities in the project.

Mares [8] introduce the fuzzification of not only durations of the activities, but also the structure of the network. He considers also the general case, that is, 
when all components of the network are vague simultaneously.

Gazdik [9] hints at various fuzzifications of the network; however, he does not elaborate, except for the fuzzy duration of the activity. The determination of $T_{A i}$ is according to (1). No backward pass is performed. He suggests that the critical path be found by enumerating all possible paths.

McCahon and Lee [10] perform forward and backward calculation and propose the application of the comparison method, instead of using the extended max and extended min.

Tsujimura et al. [11] consider the case where the triangular fuzzy numbers to be used in the network were supposed to be given by several experts; thus these numbers were greatly different. For computation they determine, according to some criteria, the so-called major and minor triangular fuzzy numbers for each activity time.

Nasution [16, 17] proposes a fuzzy critical path method by considering interactive fuzzy subtraction and by observing that only the non-negative part of the fuzzy numbers can have physical interpretation. Moreover, the calculation of activity slacks will also be considered.

Slyeptsov and Tyshchuk [18] present an efficient computation method of fuzzy times for late start and finish times of operations in the fuzzy network problems.

The fuzzy set theory is used to tackle problems where a source of vagueness is involved. Linguistic terms can be properly represented by the approximate reasoning of the fuzzy set theory. To effectively deal with the ambiguities involved in the process of linguistic estimate times.

Liang and Han [14] use the trapezoidal fuzzy numbers to make the fuzzy measures of activity times characterized by linguistic values, and proposed an algorithm for finding the fuzzy critical path of a project network.

\section{Fuzzy CPM}

In this paper, the fuzzy activity time, denote by $F A T_{i}$, of activity $A_{i}$ in a project network is represented by trapezoidal fuzzy number $F A T_{i}=\left(a_{i j}, b_{i j}, c_{i j}, d_{i j}\right)$, where $a_{i j}, d_{i j}$ are minimum and maximum values of assessing activity time for $A_{i}$, whereas $b_{i j}$ and $c_{i j}$ are the first quartile and third quartile of activity time for $A_{i}$. If there is only one set of four historical data, then $a_{i j}, b_{i j}, c_{i j}, d_{i j}$ can be sorted from minimum to maximum. Conversely, if one has no further information with respect to activity $A_{i}$, the fuzzy activity time $F A T_{i}=\left(a_{i j}, b_{i j}, c_{i j}, d_{i j}\right)$ can be evaluated subjectively by the decision-maker based on his/her knowledge, experience and subjective judgment.

Applying the extension principle, the extended algebraic operations of any two fuzzy activity times are expressed as:

$$
\begin{aligned}
& \text { Addition: } \\
& \qquad \begin{aligned}
F A T_{1} \oplus F A T_{2} & =\left(a_{1}, b_{1}, c_{1}, d_{1}\right) \oplus\left(a_{2}, b_{2}, c_{2}, d_{2}\right) \\
= & \left(a_{1}+a_{2}, b_{1}+b_{2}, c_{1}+c_{2}, d_{1}+d_{2}\right)
\end{aligned}
\end{aligned}
$$

\section{Subtraction:}

$$
\begin{array}{r}
F A T_{1} \Theta F A T_{2}=\left(a_{1}, b_{1}, c_{1}, d_{1}\right) \Theta\left(a_{2}, b_{2}, c_{2}, d_{2}\right) \\
=\left(a_{1}-d_{2}, b_{1}-c_{2}, c_{1}-b_{2}, d_{1}-a_{2}\right)
\end{array}
$$

\subsection{Ranking trapezoidal fuzzy numbers}

Ranking methods are essential in fuzzy CPM (FCPM). Many methods of ranking fuzzy numbers have been proposed. However, certain shortcomings in some of those methods have been reported in the literature. For ease of implementation, a useful ranking method developed by Liang and Han [14] is utilized to tackle the ranking problems existing in fuzzy path analysis. Let $F A T_{i}=\left(a_{i j}, b_{i j}, c_{i j}, d_{i j}\right)$ be the fuzzy activity time of activity $A_{i}$.

The decision maker's risk attitude index $\beta$ can be obtained by

$\beta=\left[\sum_{i} \sum_{j} \frac{\left(b_{i j}-a_{i j}\right)}{\left(b_{i j}-a_{i j}\right)+\left(d_{i j}-c_{i j}\right)}\right] / t$

Where $A C T$ and $t$ denote the set of all activities and the number of activities in an aircraft jet engine pump and turbine overhaul ground operation network, respectively.

For a fuzzy number $A_{i}$ with membership functions $f_{A_{i}}(x)$ we define

$m_{i}=\min \left\{x \mid f_{A_{i}}(x)=1\right\}+\max \left\{x \mid f_{A_{i}}(x)=1\right\}$. 
Now, we rank the fuzzy numbers $A_{i}$ and $A_{j}$ according to the following rules:

$A_{i}>A_{j} \Leftrightarrow R\left(A_{i}\right)>R\left(A_{j}\right), o r$,

$R\left(A_{i}\right)=R\left(A_{j}\right)$ and $m_{i}>m_{j}$,

$A_{i}=A_{j} \Leftrightarrow R\left(A_{i}\right)=R\left(A_{j}\right)$ and $m_{i}=m_{j}$

Then, the ranking value $R\left(A_{i}\right)$ of the trapezoidal fuzzy number $A i$ can be obtained as follows:

$R\left(A_{i}\right)=\beta\left[\frac{\left(d_{i}-x_{1}\right)}{\left(x_{2}-x_{1}-c_{i}+d_{i}\right)}\right]+(1-\beta)\left[1-\frac{\left(x_{2}-a_{i}\right)}{\left(x_{2}-x_{1}+b_{i}-a_{i}\right)}\right]$

Where $\beta$ is the decision maker's risk attitude index, $x_{1}=\min \left\{a_{1}, a_{2}, . ., a_{n}\right\}$, and $x_{2}=\max \left\{d_{1}, d_{2}, \ldots, d_{n}\right\}$.

By using Eq. (9) and taking the $\beta$ value calculated by Eq. (13), one can easily calculate the ranking values of $n$ trapezoidal fuzzy numbers. Based on the ranking rules described above, the ranking of $n$ trapezoidal fuzzy numbers can then be effectively determined.

\subsection{Fuzzy Critical Path Method}

We will first give the notation:

$N$ : The set of all nodes in a project network.

$A_{i}:$ The activity node $i$.

$F A T_{i}$ : The fuzzy activity time of $A_{i}$.

$F E S T_{j}$ : The earliest start fuzzy time of node $j$.

$F E C T_{j}$ : The earliest completion fuzzy time of node $j$.

$F L S T_{j}$ : The latest start fuzzy time of node $j$.

$F L C T_{j}$ : The latest completion fuzzy time of node $j$.

$F T S_{i}$ : The total slack fuzzy time of $A_{i}$.

$S(j)$ : The set of all successor activities of node $j$.

$N S(j)$ : The set of all nodes connected to all successor activities of node $j, \quad$ i.e., $N S(j)=\left\{k \mid A_{j k} \in S(j), k \in N\right\}$.

$P(j)$ : The set of all predecessor activities of node $j$.

$N P(j)$ : The set of all nodes connected to all predecessor activities of node $j$, i.e., $N P(j)=\left\{i \mid A_{i j} \in P(j), i \in N\right\}$.

$P T_{i}$ : The $i$ th path.

$P T$ : The set of all paths in a project network.
$\operatorname{FCPM}\left(P_{k}\right)$ : The total slack fuzzy time of path $P_{k}$ in a project network. The important properties of FCPM are as follows. First we set the initial node to zero for starting, i.e., $F E S T_{1}=(0,0,0,0)$. Then, the following properties are true.

\section{Property 1}

1.a.)

FEST

$=\max \left\{F E S T_{i} \oplus F A T_{i j} \mid i \in N P(j), j \neq 1, j \in N\right\}$

1.b.) $F E C T_{i}=F E S T_{i} \oplus F A T_{i}$

\section{Property 2}

2.a.)

$F L C T_{j}$

$=\min \left\{F L C T_{k} \Theta F A T_{j k} \mid k \in N S(j), j \neq n, j \in N\right\}$

2.b.) $F L S T_{j}=F L C T_{j} \Theta F A T_{j}$

\section{Property 3}

$F T S_{i j}$

$=F L C T_{j} \Theta\left(F E S T_{i} \oplus F A T_{i j}\right), 1 \leq i<j \leq n ; i, j \in N$

$F T S_{j}=F L C T_{j} \Theta F E C T_{j}=F L S T_{j} \Theta F E S T_{j}$

\section{Property 4}

$\operatorname{FCPM}\left(P_{K}\right)=\sum_{\substack{1 \leq i<j \leq n \\ i, j \in P_{K}}} F T S_{i j}, \quad P_{K} \in P T$

In an aircraft maintenance planning project network, a path $P_{C}$ such that

$$
\operatorname{FCPM}\left(P_{C}\right)=\min \left\{F C P M\left(P T_{i}\right) \mid P T_{i} \in P T\right\}
$$

is a fuzzy critical path. Thus, the theorem stated as below is true.

Theorem 1. Assume that the fuzzy activity times of all activities in an aircraft maintenance planning project network are trapezoidal fuzzy numbers; then there exists a fuzzy critical path in the network.

\subsection{Algorithm}

In this section, a fuzzy critical path algorithm is utilized to find a critical path of an aircraft maintenance 
planning project network in a fuzzy environment. The description of the algorithm is presented in the following.

\section{Fuzzy critical path algorithm:}

Step 1. Identify activities in an aircraft maintenance planning project.

Step 2. Establish precedence relationships of all activities.

Step 3. Estimate the fuzzy activity time with respect to each activity.

Step 4. Construct the aircraft maintenance planning project network.

Step 5. Let $F E S T_{1}=(0,0,0,0)$ and calculate $F E S T_{j}, \mathrm{j}$ $=2,3, \ldots, n$, by using property 1 .a.

Step 6. Let $F L C T_{n}=F E S T_{n}$ and calculate $F L C T_{j}, j=n$

$-1, n-2, \ldots, 2,1$, by using property 2 .a.

Step 7. Calculate $F T S_{j}$ with respect to each activity in an aircraft maintenance planning project network by using property 3 .

Step 8. Find all the possible paths and calculate $\operatorname{FCPM}\left(P_{k}\right)$ by using property 4 .

Step 9 . Find the fuzzy critical path by using theorem 1 .

Step 10. Find the grade of membership so that the aircraft maintenance planning project can be completed at the scheduled time. For the FCPM algorithm flow chart sees the appendix.

\section{An Application}

Modern military aircraft turbine engines present new and increasingly difficult management, operations, and maintenance problems as their levels of operating performance, complexity, and cost continue to escalate.

Aircraft Gas Turbine Engine Repair/Overhaul Technicians are employed for repairing and overhauling gas turbine engines; rebuilding gas turbine engines; balancing components and assemblies; testing and troubleshooting gas turbine engines and; inspecting gas turbine engine components and assemblies.

In some companies, the job of the gas turbine engine repair and overhaul technician is broken down into three roles, namely: inspection, disassembly/assembly and testing. The scope of the analysis, therefore, may be divided into multi-level skills depending upon company policies which would result in graduates being required to perform only a portion of the tasks listed in the analysis, while in other instances they would perform all of them.
This occupation occurs primarily in power plant shops of larger airlines, independent repair and overhaul shops and the repair and overhaul shops of engine manufacturers. Gas turbine technicians perform the disassembly, inspection, repair, assembly and testing of gas turbine engines in a clean shop environment with regular working hours. This aircraft engine specialist is required to troubleshoot problems and inspect gas turbine engine components and assemblies. This can include tearing down and rebuilding engines, balancing of components in these sophisticated, modern engines. Gas turbine engines are used in jet aircraft and power turbo-prop aircraft.

Engine overhaul takes place in specialized shops where engines are taken apart, problems diagnosed, the engine components are cleaned, repaired or rebuilt. The engine is then put back together and tested.

Tolerances and performance parameters for aircraft gas turbine engines are very high and require skilled technicians who have a strong mechanical aptitude. This job requires workers to be able to interpret technical manuals and drawings. Engine repair facilities have installed materials management data bases, and keyboarding skills are becoming more important.

The illustrative project steps for installation are determined to be as shown in Table 1. Any maintenance project consists of number of activities. These activities are represented in a network by nodes. When depicting activities on an Activity-On-Node or CPM networks the activities and their duration are shown inside the node.

We use in an AON project network; the nodes of the network are used to represent activities. Therefore, the project defined in Table 1 and all of the durations are in man-hours when represented by an Activity on- Node network yields the diagram shown in Figure 1.

There are a number of different conventions for depicting the critical path on CPM networks. By using Eq. (9), the total risk index $\beta$ is equal to 0.5215 .

Step 1.a. Forward Calculation. We now compute the $F E S T_{j}$ 's for illustrative example network. We begin by observing that set $F E S T_{A}=(0,0,0,0)$, calculate $F E S T_{j}=\max \left\{F E S T_{i} \oplus F A T_{i j} \mid i \in N P(j), j \neq 1, j \in N\right\}$, $\mathrm{j}=\mathrm{B}, \mathrm{C}, \mathrm{D}, \ldots \mathrm{X}$ by using property 1 .a. since node $\mathrm{A}$ (Node 1) is the only immediate predecessors of node B, $\mathrm{C}$ and D. Thus 
Table 1. The fuzzy activity time in the aircraft gas turbine engine repair/overhaul operations network shown as Figure 1

\begin{tabular}{|c|c|c|c|c|c|}
\hline \multicolumn{2}{|c|}{ No.Code } & \multirow[t]{2}{*}{ Activity Description } & \multirow{2}{*}{$\begin{array}{c}\begin{array}{c}\text { Precedence } \\
\text { Activity }\end{array} \\
-\end{array}$} & \multirow{2}{*}{$\begin{array}{l}\text { Fuzzy activity time } \\
\text { Around } 32 \text { man-hours }\end{array}$} & \multirow{2}{*}{$\begin{array}{c}F A T_{i} \\
(28,32,32,36)\end{array}$} \\
\hline 1 & A & & & & \\
\hline 2 & $\mathrm{~B}$ & Calibrate all ganges & A & Around 80 man-hours & $(72,80,80,88)$ \\
\hline 3 & $\mathrm{C}$ & Dismantle pump cover and remove rotor & A & Around 8 man-hours & $(6,8,8,10)$ \\
\hline 4 & $\mathrm{D}$ & Dismantle turbine cover and remove rotor & A & Around 16 man-hours & $(12,16,16,20)$ \\
\hline 5 & $\mathrm{E}$ & Clean all ganges and line & B & Around 32 man-hours & $(28,32,32,36)$ \\
\hline 6 & $\mathrm{~F}$ & Replace ganges & $\mathrm{E}$ & Around 16 man-hours & $(12,16,16,20)$ \\
\hline 7 & $\mathrm{G}$ & Repair lubrication system & $\mathrm{C}$ & Around between 28 and 40 man-hours & $(28,32,36,40)$ \\
\hline 8 & $\mathrm{H}$ & Rebuild impeller & $\mathrm{C}$ & Around between 256 and 352 man-hours & $(256,288,320,352)$ \\
\hline 9 & I & Clean pump casting & $\mathrm{C}$ & Around 32 man-hours & $(24,32,32,36)$ \\
\hline 10 & $\mathrm{~J}$ & Fix pump bearings & G & Around 16 man-hours & $(12,16,16,20)$ \\
\hline 11 & $\mathrm{~K}$ & Balance Impeller & $\mathrm{H}$ & Around 32 man-hours & $(28,32,32,36)$ \\
\hline 12 & $\mathrm{~L}$ & Reinstall Impeller & $\mathrm{I}, \mathrm{J}, \mathrm{K}$ & Around 16 man-hours & $(12,16,16,20)$ \\
\hline 13 & $\mathrm{M}$ & Rebuild turbine rotor & $\mathrm{D}$ & Around between 260 and 400 man-hours & $(260,320,360,400)$ \\
\hline 14 & $\mathrm{~N}$ & Check turbine bearings & $\mathrm{D}$ & Around 16 man-hours & $(10,16,16,18)$ \\
\hline 15 & $\mathrm{O}$ & Balance turbine rotor & M & Around 64 man-hours & $(56,64,64,72)$ \\
\hline 16 & $\mathrm{P}$ & Fix turbine bearings & $\mathrm{N}$ & Around 16 man-hours & $(12,16,16,20)$ \\
\hline 17 & Q & Fix turbine rotor & $\mathrm{O}, \mathrm{P}$ & Around between 16 and 28 man-hours & $(16,20,24,28)$ \\
\hline 18 & $\mathrm{R}$ & Fix turbine cover & Q & Around between 20 and 32 man-hours & $(20,24,28,32)$ \\
\hline 19 & $\mathrm{~S}$ & Test components & $\mathrm{R}$ & Around between 12 and 18 man-hours & $(12,14,16,18)$ \\
\hline 20 & $\mathrm{~T}$ & Check clearance & $\mathrm{R}$ & Around 16 man-hours & $(14,16,16,18)$ \\
\hline 21 & $\mathrm{U}$ & Fix pump bearings & $\mathrm{L}$ & Around 16 man-hours & $(12,16,16,20)$ \\
\hline 22 & $\mathrm{~V}$ & Fix pump cover & $\mathrm{U}$ & Around between 18 and 24 man-hours & $(18,20,22,24)$ \\
\hline & & Install shaft packing & V & Around 16 man-hours & $(14,16,16,18)$ \\
\hline 24 & $\mathrm{X}$ & Final test & $\mathrm{S}, \mathrm{T}, \mathrm{W}, \mathrm{F}$ & Around between 48 and 72 man-hours & $(48,56,64,72)$ \\
\hline
\end{tabular}

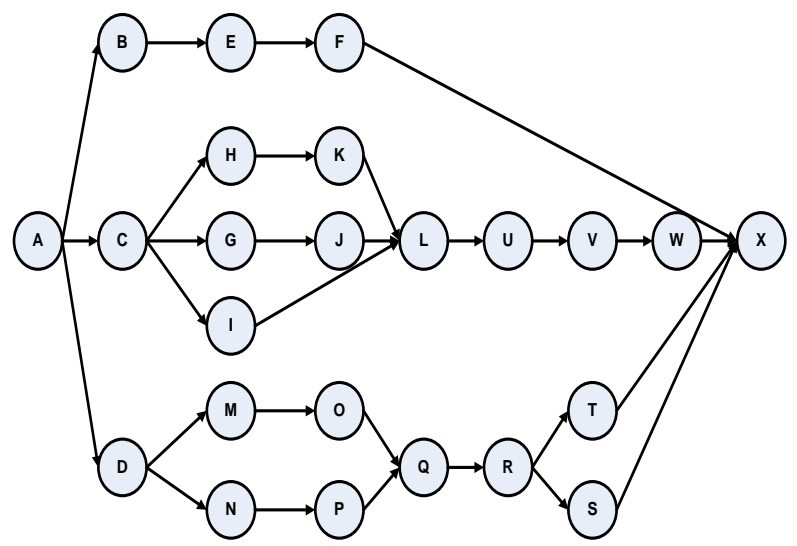

Figure 1. Network diagram

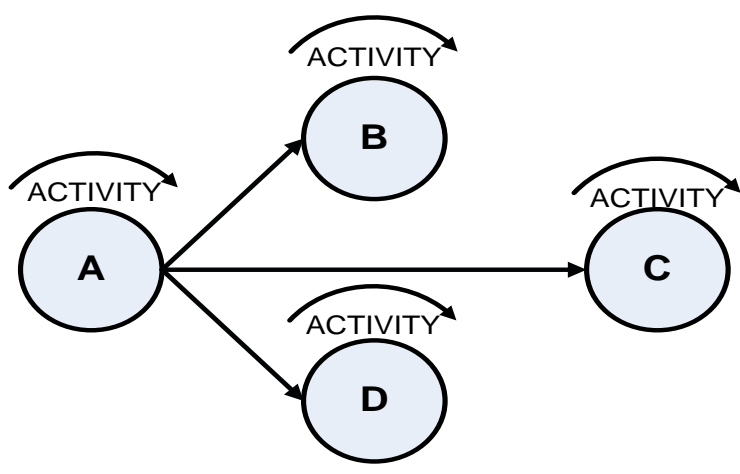

Figure 2. B, C and D cannot begin until A has been completed. 


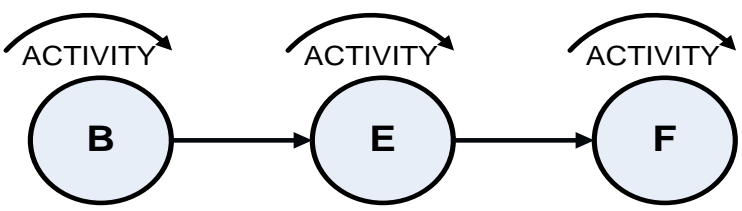

Figure 3. Simple precedence B precedence E, which precedence $\mathrm{F}$.

For Figure 2:

$F E S T_{B}=F E S T_{A} \oplus F A T_{A}=(0,0,0,0) \oplus(28,32,32,36)=(28,32,32,36)$ $F E S T_{C}=F_{E S T_{A}} \oplus F A T_{A}=(0,0,0,0) \oplus(28,32,32,36)=(28,32,32,36)$ $F E S T_{D}=F E S T_{A} \oplus F A T_{A}=(0,0,0,0) \oplus(28,32,32,36)=(28,32,32,36)$

For Figure 3 :

$F E S T_{E}=F E S T_{B} \oplus F A T_{B}=(28,32,32,36) \oplus(72,80,80,88)=(100,112,112,124)$ $F E S T_{F}=F E S T_{E} \oplus F A T_{E}=(100,112,112,124) \oplus(28,32,32,36)=(128,144,144,160)$

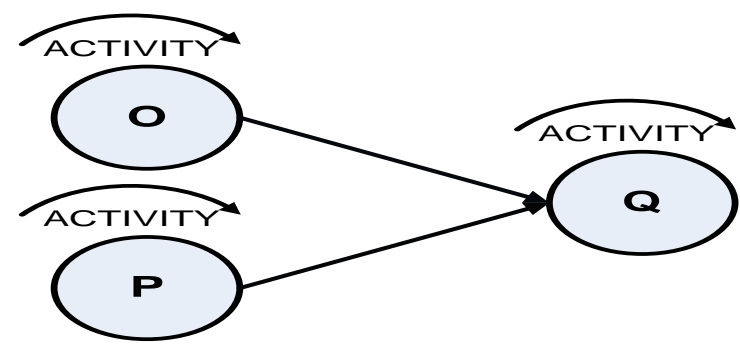

Figure 4. Activity $\mathrm{Q}$ cannot take place until activities $\mathrm{O}$ and $\mathrm{P}$ are completed.

$$
\begin{aligned}
& F E S T_{Q}=\max \left\{\left[F E S T_{O} \oplus F A T_{O}\right],\left[F E S T_{P} \oplus F A T_{P}\right]\right\} \\
& \left.\begin{array}{l}
=\max \left\{\begin{array}{l}
{[(320,368,408,456) \oplus(56,64,64,72)],} \\
{[(54,64,64,74) \oplus(12,16,16,20)]}
\end{array}\right\} \text { for Figure } 4 . \\
=\max \{(376,432,472,528),(66,80,80,94)\}
\end{array}\right\}
\end{aligned}
$$

by Eq. (13) and taking $\beta=0.5215$, the ranking value of $(376,432,472,528)$ and $(66,80,80,94)$ can be obtained: $\quad x_{1}=\min \left\{a_{1}, a_{2}, . ., a_{n}\right\}=\min (376,66)=66$, $x_{2}=\max \left\{d_{1}, d_{2}, \ldots, d_{n}\right\}=\max \{528,94\}=528$,

$$
\begin{aligned}
& \{(376,432,472,528),(66,80,80,94)\} \\
& x_{1}=66, x_{2}=528 \\
& R((376,432,472,528))= \\
& 0.5215\left[\frac{(528-66)}{(528-66-472+528)}\right] \\
& +(1-0.5215)\left[1-\frac{(528-376)}{(528-62+432-376)}\right]=0.804 \\
& R((66,80,80,94))=0.5215\left[\frac{(94-66)}{(528-66-80+94)}\right] \\
& +(1-0.5215)\left[1-\frac{(528-66)}{(528-66+80-62)}\right]=0.0486
\end{aligned}
$$

Since $R((376,432,472,528))>R((66,80,80,94))$, $F E S T_{Q}=(376,432,472,528)$. And go on.

Step 1.b. Calculate $F E C T_{i}=F E S T_{i} \oplus F A T_{i}$ by using property 1.b.

$$
\begin{aligned}
& F E C T_{A}=F E S T_{A} \oplus F A T_{A} \\
& =(0,0,0,0) \oplus(28,32,32,36) \\
& =(28,32,32,36) \\
& F E C T_{B}=F E S T_{B} \oplus F A T_{B} \\
& =(28,32,32,36) \oplus(72,80,80,88) \\
& =(100,112,112,124) \\
& \ldots \\
& \ldots \\
& F E C T_{O}=F E S T_{O} \oplus F A T_{O} \\
& =(320,368,408,456) \oplus(56,64,64,72) \\
& =(376,432,472,528)
\end{aligned}
$$

$F E C T_{P}=F E S T_{P} \oplus F A T_{P}$

$=(128,144,144,160) \oplus(12,16,16,20)$

$=(140,160,160,180)$

$F E C T_{Q}=F E S T_{Q} \oplus F A T_{Q}$

$=(376,432,472,528) \oplus(16,20,24,28)$

$=(392,452,496,556)$ 
Step 2.a Backward calculation:

Set $F L C T_{X}=(474,548,604,678)$ and calculate $F L C T_{j}, j=$ $\mathrm{W}, \mathrm{V}, \ldots, \mathrm{B}, \mathrm{A}$ by using property 2 .

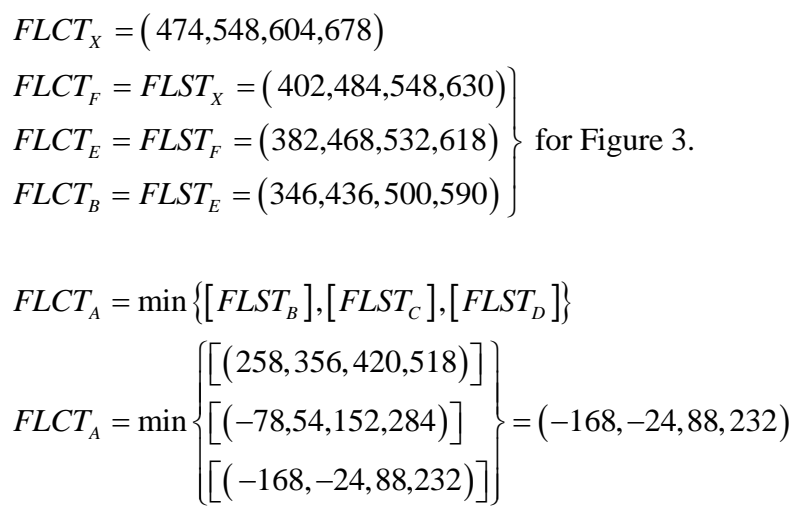

by Eq. (13) and taking $\beta=0.5215$, the ranking value of $(258,356,420,518),(-78,54,152,284)$ and

$(-168,-24,88,232)$ can be obtained: min value of the ranking value is $F L C T_{A}=(-168,-24,88,232)$.

Step 2.b. Calculate $F L S T_{j}=F L C T_{j} \Theta F A T_{j}$ by using property $1 . b$.

$$
\begin{aligned}
& F L S T_{j}=F L C T_{j} \Theta F A T_{j} \\
& F L S T_{X}=F L C T_{X} \Theta F A T_{X} \\
& =(474,548,604,678) \Theta(48,56,64,72) \\
& =(402,484,548,630) \\
& F L S T_{F}=F L C T_{F} \Theta F A T_{F} \\
& =(402,484,548,630) \Theta(12,16,16,20) \\
& =(382,468,532,618) \\
& \ldots \\
& F L S T_{A}=F L C T_{A} \Theta F A T_{A} \\
& =(-168,-24,88,232) \Theta(28,32,32,36) \\
& =(-204,-56,56,204)
\end{aligned}
$$

Step 3. If computing all parameters of fuzzy CPM we can calculate $F T S_{j}$ with respect to each activity by property 3 .
Property 3

$$
\begin{aligned}
& F T S_{j}=F L C T_{j} \Theta F E C T_{j}=F L S T_{j} \Theta F E S T_{j} \\
& F T S_{A}=F L C T_{A} \Theta F E C T_{A}=(-168,-24,88,232) \Theta(28,32,32,36) \\
& =(-204,-56,56,204) \\
& F T S_{B}=F L C T_{B} \Theta F E C T_{B}=(346,436,500,590) \Theta(100,112,112,114) \\
& =(222,324,388,490) \\
& \ldots \\
& F T S_{X}=F L C T_{X} \Theta F E C T_{X} \\
& =(474,548,604,678) \Theta(454,548,604,678) \\
& =(-204,-56,56,204)
\end{aligned}
$$

Step 4. Find all the possible paths and calculate $\operatorname{FCPM}\left(P_{k}\right)$ by using property 4 .

$P=\left\{\begin{array}{l}(\text { A-B-E-F-X }),(\text { A-C-H-K-L-U-V-W-X }), \\ (\text { A-C-G-J-L-U-V-W-X), (A-C-I-L-U-V-W-X }) \\ (\text { A-D-M-O-Q-R-T-X), (A-D-M-O-Q-R-S-X), } \\ (\text { A-D-N-P-Q-R-T-X), (A-D-N-P-Q-R-S-X) }\end{array}\right\}$

Let path $P_{1}=(\mathrm{A}-\mathrm{B}-\mathrm{E}-\mathrm{F}-\mathrm{X})$, then

$F C P M\left(P_{1}\right)=F T S_{A} \oplus F T S_{B} \oplus F T S_{E} \oplus F T S_{F} \oplus F T S_{X}$ $\operatorname{FCPM}\left(P_{1}\right)=\left[\left(\begin{array}{llll}-204 & -56 & 56 & 204\end{array}\right) \oplus(222324388\right.$ 490 $)$

$\left.\oplus(222324388490) \oplus\left(\begin{array}{llll}222 & 324 & 388 & 490\end{array}\right) \oplus\left(\begin{array}{llll}-204 & -56 & 56 & 204\end{array}\right)\right]$ $\operatorname{FCPM}\left(P_{1}\right)=(258,860,1276,1878)$

then go on.

Step 5. Find the fuzzy critical path by using theorem 1 and taking $\beta=0.5215$, the ranking value of $\operatorname{FCPM}\left(P_{k}\right), i=1,2, \ldots, 8$ can be obtained in Table 2 :

Since $\begin{array}{ll}R\left(F C P M\left(P_{5}\right)\right)<R\left(F C P M\left(P_{6}\right)\right)<R\left(F C P M\left(P_{2}\right)\right) \\ <R\left(F C P M\left(P_{4}\right)\right)<R\left(F C P M\left(P_{7}\right)\right)<R\left(F C P M\left(P_{8}\right)\right)\end{array}$, $<R\left(F C P M\left(P_{1}\right)\right)<R\left(F C P M\left(P_{3}\right)\right)$, the fuzzy critical path is $P_{5}$, (A-D-M-O-Q-R-T-X) and the Aircraft Gas Turbine Engine Repair/Overhaul operations completion time is approximately between 548 and 604 man-hours, i.e., $(474,548,604,678)$. Further, the grade of membership that the aircraft engine maintenance operation completed time can be obtained. For example, the grade of membership that the aircraft engine maintenance operation completion time being within 500 minutes is 0.489 . As it can be seen from this analysis, the path where aircraft engine maintenance operations influence combination workers is $P_{5}$. 
Table 2. FCPM ranking values

\begin{tabular}{lllllllll}
\hline No. & For & RFCPM & RFCPM & Total FTS & & \multicolumn{3}{c}{ Order } \\
\hline 1 & A-B-E-F-X & $R\left(F C P M\left(P_{1}\right)\right)$ & 0.5992 & 258 & 860 & 1276 & 1878 & 7 \\
2 & A-C-H-K-L-U-V-W-X & $R\left(F C P M\left(P_{2}\right)\right)$ & 0.4857 & -1206 & 42 & 952 & 2200 & 3 \\
3 & A-C-G-J-L-U-V-W-X & $R\left(F C P M\left(P_{3}\right)\right)$ & 0.6315 & -550 & 642 & 1496 & 2688 & 8 \\
4 & A-C-I-L-U-V-W-X & $R\left(F C P M\left(P_{4}\right)\right)$ & 0.5678 & -740 & 340 & 1120 & 2200 & 6 \\
5 & A-D-M-O-Q-R-T-X & $R\left(F C P M\left(P_{5}\right)\right)$ & 0.3864 & -1632 & -448 & 448 & 1632 & 1 \\
6 & A-D-M-O-Q-R-S-X & $R\left(F C P M\left(P_{6}\right)\right)$ & 0.3867 & -1632 & -448 & 450 & 1634 & 2 \\
7 & A-D-N-P-Q-R-T-X & $R\left(F C P M\left(P_{7}\right)\right)$ & 0.5757 & -764 & 336 & 1152 & 2252 & 4 \\
8 & A-D-N-P-Q-R-S-X & $R\left(F C P M\left(P_{8}\right)\right)$ & 0.5760 & -764 & 336 & 1154 & 2254 & 5 \\
\hline
\end{tabular}

If workers are assigned through this path for maintenance service operations to maintenance by authorization, the average maintenance operations time wasted will be at its longest. This is to say that if the efficiency of aircraft engine maintenance services operations is to be raised; improving the efficiency obtained from these processes needs to be done.

In this paper a new method has been presented for solving the project scheduling problem in the fuzzy environment. In this paper we considered that the durations of activities in a project are positive trapezoidal fuzzy numbers and for first time it is assumed that the relationship between activities are fuzzy numbers.

Previous researches have proposed some models for solving project scheduling problem in the fuzzy environment. Our proposed method calculates all parameters of project such as earliest and latest start and finish time and slack times. We show the calculated values of these parameters for our problem as in the next page Table 3 .

\section{Conclusions}

If an aircraft maintenance operations and service system is inefficient, or if the operational procedures are ineffective, these factors can contribute to flight delays for airlines which may prove very costly in a long-term. This highlights the importance of the appropriate maintenance operational procedures and performance indicators in aircraft maintenance management. Despite this importance, there has been scant attention given to this aspect in maintenance management context.
Though there have been appeals to avoid the delays and inefficiencies experienced in airside, landside, airlines' and maintenance operations, the available operation measurement models to cut down airlines maintenance's cost only provide vague and uncertain decision-making information. An aircraft maintenance center is an interconnected set of physical facilities and components. For an aircraft maintenance center to function efficiently, the capacities of each of these elements must be matched. Relief of a bottleneck in one part of the aircraft maintenance center will not have the desired effect on improving overall through maintenance-traffic unless other parts are capable of absorbing a greater influx of maintenance-traffic. Apart from this, the goodness-of-fit test for the activity times to satisfy beta distribution is not only a troublesome process, but may yield disappointing results as well.

Hence, the conventional precision-based/ randomoriented project analysis tends to be less effective in sustaining reliable information in an imprecise and fuzzy decision environment.

This paper uses a fuzzy critical path algorithm to tackle the problem in fuzzy aircraft maintenance planning operation decision analysis. The method takes into account the rating attitude (optimistic/pessimistic) of decision makers. Thus, by conducting fuzzy or nonfuzzy activity time assessments, decision makers can automatically obtain the fuzzy critical path. Therefore, only when customs collectively moves to meet the needs of the marketplace can the aircraft maintenance service is totally improved and secure. For further research, we suggest resource constraints to be taken account in the scheduling problem. Resource constraints can be modeled using fuzzy numbers. 
Table 3. Summarized fuzzy CPM parameters as follows shown as Figure 1

\begin{tabular}{|c|c|c|c|c|c|c|c|c|c|c|c|c|c|c|c|c|c|c|c|c|c|}
\hline \multicolumn{2}{|c|}{ No.Code } & \multicolumn{4}{|c|}{ FEST } & \multicolumn{4}{|c|}{ FECT } & \multicolumn{4}{|c|}{ FLST } & \multicolumn{4}{|c|}{ FLCT } & \multicolumn{4}{|c|}{ FTS(FLCT-FECT) } \\
\hline 1 & A & 0 & 0 & 0 & 0 & 28 & 32 & & 36 & -204 & -56 & 56 & 204 & -168 & -24 & 88 & 232 & -204 & -56 & 56 & $6 \quad 204$ \\
\hline 2 & $\mathrm{~B}$ & 28 & 32 & 32 & 36 & 100 & 112 & 112 & 124 & 258 & 356 & 420 & 518 & 346 & 436 & 500 & 590 & 222 & 324 & 388 & 88490 \\
\hline 3 & $\mathrm{C}$ & 28 & 32 & 32 & 36 & 34 & 40 & 40 & 46 & -78 & 54 & 152 & 284 & -68 & 62 & 160 & 290 & -114 & 22 & 120 & 20256 \\
\hline 4 & $\mathrm{D}$ & 28 & 32 & 32 & 36 & 40 & 48 & 48 & 56 & -168 & -24 & 88 & 232 & -148 & -8 & 104 & 244 & -204 & -56 & 56 & $6 \quad 204$ \\
\hline 5 & $\mathrm{E}$ & 100 & 112 & 112 & 124 & 128 & 144 & 144 & 160 & 346 & 436 & 500 & 590 & 382 & 468 & 532 & 618 & 222 & 324 & 388 & $88 \quad 490$ \\
\hline 6 & $\mathrm{~F}$ & 128 & 144 & 144 & 160 & 140 & 160 & 160 & 180 & 382 & 468 & 532 & 618 & 402 & 484 & 548 & 630 & 222 & 324 & 388 & $88 \quad 490$ \\
\hline 7 & $\mathrm{G}$ & 3 & 40 & 40 & 46 & 62 & 72 & 76 & 86 & 260 & 362 & 432 & 534 & 300 & 398 & 464 & 562 & 214 & 322 & 392 & 92500 \\
\hline 8 & $\mathrm{H}$ & 3 & 40 & 40 & 46 & 290 & 328 & 360 & 398 & -68 & 62 & 160 & 290 & 284 & 382 & 448 & 546 & -114 & 22 & 120 & 20256 \\
\hline 9 & I & 3 & 40 & 40 & 46 & 62 & 72 & 72 & 82 & 284 & 382 & 448 & 546 & 320 & 414 & 480 & 574 & 238 & 342 & 408 & $08 \quad 512$ \\
\hline 10 & $\mathrm{~J}$ & 6 & 72 & 76 & 86 & 74 & 88 & 92 & 106 & 300 & 398 & 464 & 562 & 320 & 414 & 480 & 574 & 214 & 322 & 392 & 92500 \\
\hline 11 & $\mathrm{~K}$ & 290 & 328 & 360 & 398 & 318 & 360 & 392 & 434 & 284 & 382 & 448 & 546 & 320 & 414 & 480 & 574 & -114 & 22 & 120 & 20256 \\
\hline 12 & $\mathrm{~L}$ & 318 & 360 & 392 & 434 & 330 & 376 & 408 & 454 & 320 & 414 & 480 & 574 & 340 & 430 & 496 & 586 & -114 & 22 & 120 & 20256 \\
\hline 13 & M & 40 & 48 & 48 & 56 & 320 & 368 & 408 & 456 & -148 & -8 & 104 & 244 & 252 & 352 & 424 & 524 & -204 & -56 & 56 & $6 \quad 204$ \\
\hline 14 & $\mathrm{~N}$ & 40 & 48 & 48 & 56 & 54 & 64 & 64 & 74 & 286 & 384 & 456 & 554 & 304 & 400 & 472 & 568 & 230 & 336 & 40 & $08 \quad 514$ \\
\hline 15 & $\mathrm{O}$ & 320 & 368 & 408 & 456 & 376 & 432 & 472 & 528 & 252 & 352 & 424 & 524 & 324 & 416 & 488 & 580 & -204 & -56 & 56 & $6 \quad 204$ \\
\hline 10 & $\mathrm{P}$ & 5 & 64 & 64 & 74 & 6 & 80 & 80 & 94 & 304 & 400 & 472 & 568 & 324 & 416 & 488 & 580 & 230 & 336 & 40 & 08514 \\
\hline 17 & Q & 376 & 432 & 472 & 528 & 392 & 452 & 496 & 556 & 324 & 416 & 488 & 580 & 352 & 440 & 508 & 596 & -204 & -56 & 56 & $6 \quad 204$ \\
\hline 18 & $\mathrm{R}$ & 392 & 452 & 496 & 556 & 412 & 476 & 524 & 588 & 352 & 440 & 508 & 596 & 384 & 468 & 532 & 616 & -204 & -56 & 56 & $6 \quad 204$ \\
\hline 19 & $\mathrm{~S}$ & 412 & 476 & 524 & 588 & 424 & 490 & 540 & 606 & 384 & 468 & 534 & 618 & 402 & 484 & 548 & 630 & -204 & -56 & 58 & $8 \quad 206$ \\
\hline 20 & $\mathrm{~T}$ & 412 & 476 & 524 & 588 & 426 & 492 & 540 & 606 & 384 & 468 & 532 & 616 & 402 & 484 & 548 & 630 & -204 & -56 & 56 & $6 \quad 204$ \\
\hline 21 & $\mathrm{U}$ & 330 & 376 & 408 & 454 & 342 & 392 & 424 & 474 & 340 & 430 & 496 & 586 & 360 & 446 & 512 & 598 & -114 & 22 & 12 & 20256 \\
\hline 22 & $\mathrm{~V}$ & 342 & 392 & 424 & 474 & 360 & 412 & 446 & 498 & 360 & 446 & 512 & 598 & 384 & 468 & 532 & 616 & -114 & 22 & 12 & 20256 \\
\hline 23 & W & 360 & 412 & 446 & 498 & 374 & 428 & 462 & 516 & 384 & 468 & 532 & 616 & 402 & 484 & 548 & 630 & -114 & 22 & 12 & 20256 \\
\hline 24 & $X$ & 426 & 492 & 540 & 606 & 474 & 548 & 604 & 678 & 402 & 484 & 548 & 630 & 474 & 548 & 604 & 678 & -204 & -56 & 56 & $6 \quad 204$ \\
\hline
\end{tabular}




\section{References}

1. D. Dubois and H. Prade, Fuzzy Sets and Systems: Theory and Applications (Academic Press, 1980).

2. D. Dubois and H. Prade, In Possibility Theory: An Approach to Computerized (Processing of Uncertainty, Plenum, New York), (1988) 62-63.

3. D. Dubois and H. Prade, Decision making under fuzziness. In Advances in Fuzzy Set Theory, (NorthHolland, Amsterdam), (1979) 279-302.

4. M. Mizumoto and K. Tanaka, Some properties of fuzzy numbers. In Advances in Fuzzy Set Theory, (NorthHolland, Amsterdam), (1979) 153-164.

5. S. Chanas and J. Kamburowski, The use of fuzzy variables in PERT. Fuzzy Sets and Systems 5 (1981) 1119.

6. S. Chanas, Fuzzy sets in few classical operational research problems. In Approximate Reasoning in Decision Analysis, (North-Holland, and Amsterdam, 1982), 351-363.

7. J.J. Buckley, Fuzzy PERT. In Applications of Fuzzy Set Methodologies in Industrial Engineering, (Amsterdam, 1989), 103-114.

8. M. Mares, Network analysis of fuzzy technologies. In Applications of Fuzzy Set Methodologies in Industrial Engineering, (Elsevier, Amsterdam, 1989), 115-125.

9. I. Gazdik, Fuzzy network planning. IEEE Trans. Reliability 32 August (1983) 304-313.

10. C.S. McCahon and E.S. Lee, Project network analysis with fuzzy activity times. Comput. Math. Appl. 15(10) (1988) 829-838.

11. Y. Tsujimura, I.S. Chang and M. Gen, An effective method for large scale project planning with multiple fuzzy activity times. In Second IEEE International Conference on Fuzzy Systems, (San Francisco, 1993) 1009-1015.

12. S. Chanas S and P. Zielinski, Critical Path Analysis in the Network with Fuzzy Activity Times, Fuzzy Sets and Systems, Vol. 122, (2001) 195-204.

13. T.C. Han, C.C. Chung and G.S. Liang, Application of Fuzzy Critical Path Method to Airport's Cargo Ground Operation Systems, Journal of Marine Science and Technology, Vol. 14, No. 3, (2006) 139-146.

14. G.S. Liang and T.C. Han., Critical Path Analysis Based on Fuzzy Concept, International Journal of Information and Management Sciences, Vol. 15, No. 4, (2004) 29-40.

15. S. Chen and Y. Hsueh, A simple approach to fuzzy critical path analysis in project Networks, Applied Mathematical Modeling 32 (2008) 1289-1297.

16. S.H. Nasution, Fuzzy durations in critical path method. In Second IEEE International Conference on Fuzzy Systems, (San Francisco, 1993) 1069-1073.

17. S.H. Nasution, Fuzzy Critical Path Method, IEEE Transportation Systems Man Cybernet, Vol. 24, (1994) 48-57.
18. A.I. Slyeptsov and T.A. Tyshchuk, A Method of Computation of Characteristics of Operations in a Problem of Fuzzy Network, Cybernetics and Planning and Management System Analysis, Vol. 39, No. 3, (2003) 367-378

19. I. Ertugrul and N. Karakasoglu, Fuzzy Pert and its Application to Machine Installation Process Journal of Multiple-Valued Logic and Soft Computing, Volume 15, Number 1, (2009).

20. O. Atli, Tabu Search and an Exact Algorithm for the Solutions of Resource-constrained Project Scheduling Problems, International Journal of Computational Intelligence Systems Vol.4, (2011) pp. 255 - 267.

\section{Appendix}

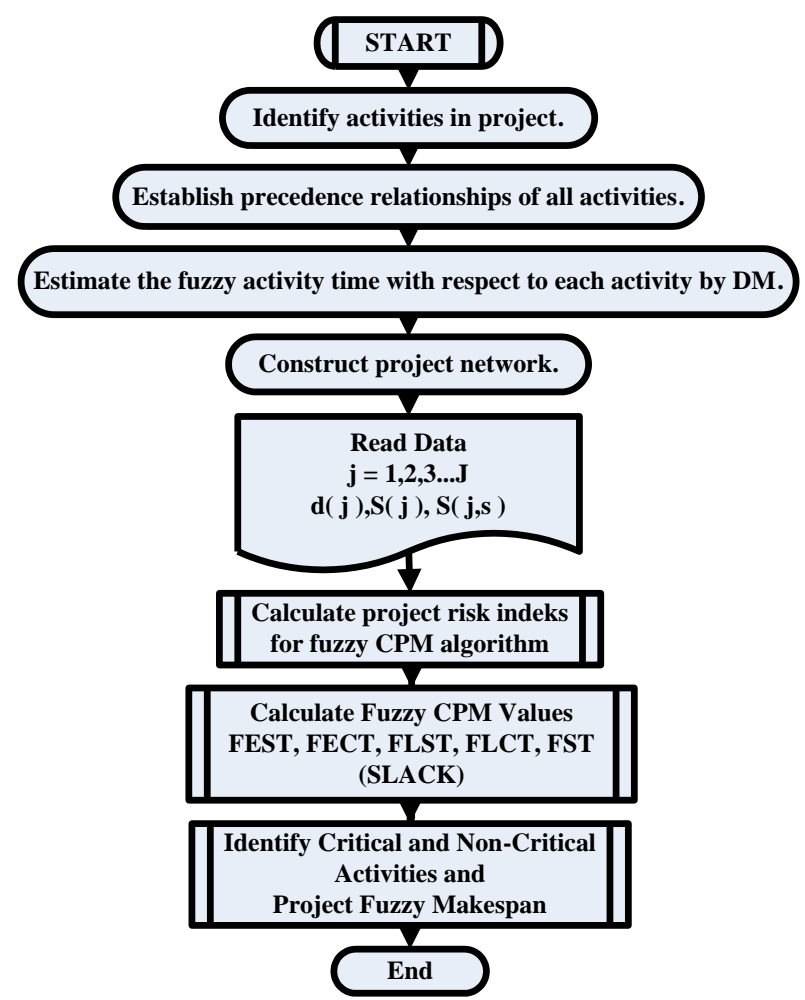

Flow Chart of the FCPM Algorithm 